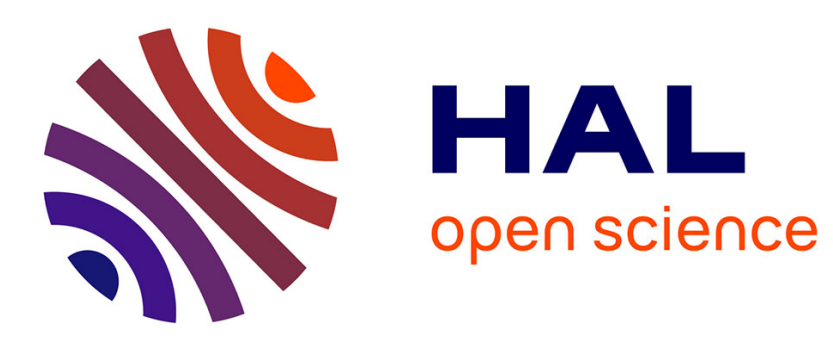

\title{
Experimental evaluation of magnified haptic feedback for robot-assisted needle insertion and palpation
}

\author{
Leonardo Meli, Claudio Pacchierotti, Domenico Prattichizzo
}

\section{To cite this version:}

Leonardo Meli, Claudio Pacchierotti, Domenico Prattichizzo. Experimental evaluation of magnified haptic feedback for robot-assisted needle insertion and palpation. The International Journal of Medical Robotics and Computer Assisted Surgery, 2017, 13 (4), pp.1-14. 10.1002/rcs.1809 . hal-01482249

\author{
HAL Id: hal-01482249 \\ https://hal.inria.fr/hal-01482249
}

Submitted on 3 Mar 2017

HAL is a multi-disciplinary open access archive for the deposit and dissemination of scientific research documents, whether they are published or not. The documents may come from teaching and research institutions in France or abroad, or from public or private research centers.
L'archive ouverte pluridisciplinaire HAL, est destinée au dépôt et à la diffusion de documents scientifiques de niveau recherche, publiés ou non, émanant des établissements d'enseignement et de recherche français ou étrangers, des laboratoires publics ou privés. 


\title{
Experimental evaluation of magnified haptic feedback for robot-assisted needle insertion and palpation
}

\author{
Leonardo Meli, Claudio Pacchierotti, and Domenico Prattichizzo
}

\begin{abstract}
Background: Haptic feedback has been proven to play a key role in enhancing the performance of teleoperated medical procedures. However, due to safety issues, commerciallyavailable medical robots do not currently provide the clinician with haptic feedback.

Methods: This work presents the experimental evaluation of a teleoperation system for robot-assisted medical procedures able to provide magnified haptic feedback to the clinician. Forces registered at the operating table are magnified and provided to the clinician through a 7-DoF haptic interface. The same interface is also used to control the motion of a 6-DoF slave robotic manipulator. The safety of the system is guaranteed by a time-domain passivity-based control algorithm.

Results: We carried out two experiments on stiffness discrimination (during palpation and needle insertion) and one experiment on needle guidance.

Conclusions: Our haptic-enabled teleoperation system improved the performance with respect to direct hand interaction of $80 \%, 306 \%$, and $27 \%$ in stiffness discrimination through palpation, stiffness discrimination during needle insertion, and guidance, respectively.
\end{abstract}

Index Terms - haptics, needle insertion, telesurgery, navigation, safety, minimally-invasive surgery.

\section{INTRODUCTION}

$\mathbf{R}$ OBOT-ASSISTED surgery is increasingly becoming an accepted component of the state-of-the-art operating room. This success is due to multiple different reasons, such as increased precision, improved visualization, reduced instruments tremor, error-free and timely repetitive tasks execution, reduced incision size, and shorter hospitalization. ${ }^{1,2}$ However, there are still a few disadvantages with respect to conventional laparoscopic surgery, and several research groups are currently trying to address these limitations. Among many others, Lanfranco et al. ${ }^{1}$ and the SAGES-MIRA Robotic Surgery Consensus Group ${ }^{2}$ indicate the lack of haptic feedback as one of the main limitations of nowadays robot-assisted surgery. Indeed, haptic feedback has been proven to play a key role in enhancing the performance of teleoperated surgical procedures in a wide range of applications, including microneedle positioning, ${ }^{3,4}$ telerobotic catheter insertion, ${ }^{5}$ suturing simulation, ${ }^{6}$ palpation, ${ }^{7}$ cardiothoracic procedures, ${ }^{8}$ keyhole neurosurgery, ${ }^{9}$ endoscopic surgery, ${ }^{10}$ micromanipulation, ${ }^{11}$ and

L. Meli and D. Prattichizzo are affiliated with the Dept. of Advanced Robotics, Istituto Italiano di Tecnologia, Genova, Italy and with the Dept. of Information Engineering and Mathematics, University of Siena, Siena, Italy. E-mail: leonardo.meli, domenico.prattichizzo@iit.it.

C. Pacchierotti is affiliated with the CNRS at Irisa and Inria Rennes, Rennes, France. E-mail: claudio.pacchierotti@irisa.fr.

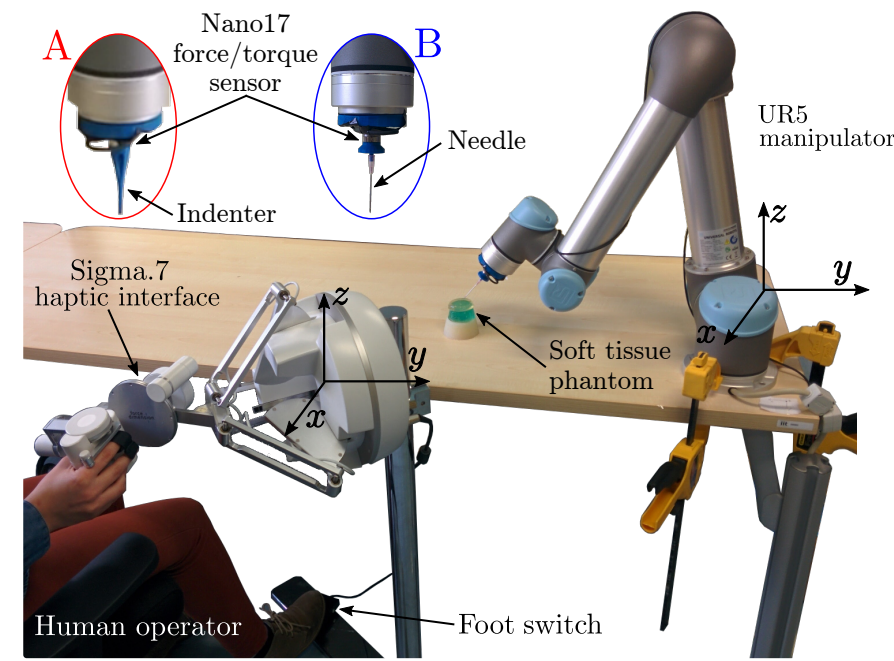

Fig. 1. Teleoperation system. The master system consists of a Sigma.7 haptic interface, while the slave system is composed of a 6-DoF force/torque sensor (ATI Nano17) mounted on a 6-DoF UR5 robotic arm. Depending on the task, we mounted two different end-effectors: (A) a spherical-ended conical indenter for palpation (used in Sec. II-B3) or (B) a 18-gauge prostate brachytherapy needle for needle insertion (used in Secs. II-B4 and II-B5).

cell injection. ${ }^{12}$ Its benefits typically include increased manipulation accuracy, ${ }^{13,14}$ increased perception accuracy, ${ }^{15-17}$ decreased completion time, ${ }^{18,19}$ and decreased peak and mean force applied to the remote environment. ${ }^{20,21}$ Among the diverse applications presented in the literature, robot-assisted needle insertion and palpation seem to particularly benefit from the addition of haptic feedback cues. For example, Kinget al. ${ }^{22,23}$ developed a modular pneumatic haptic feedback system to improve the performance of the da Vinci surgical system. The system includes piezoresistive force sensors mounted on the gripping surfaces of a robotic tool and two pneumatic balloon-array tactile displays mounted on the robot's master console. More recently, $\mathrm{Li}$ et al. ${ }^{24}$ extended this approach to three fingers, presenting a compact pneumatic system for robot-assisted palpation. It simulates tissue stiffness by changing the pressure in three balloons placed on the index, middle, and ring fingers. Stanley and Okamura ${ }^{25}$ combined pneumatics and particle jamming to simultaneously control the shape and mechanical properties of a cutaneous haptic display. The system includes a hollow silicone membrane molded into an array of thin cells. Each cell is filled with coffee grounds such that adjusting the vacuum level in any individual cell rapidly switches it between flexible and rigid 


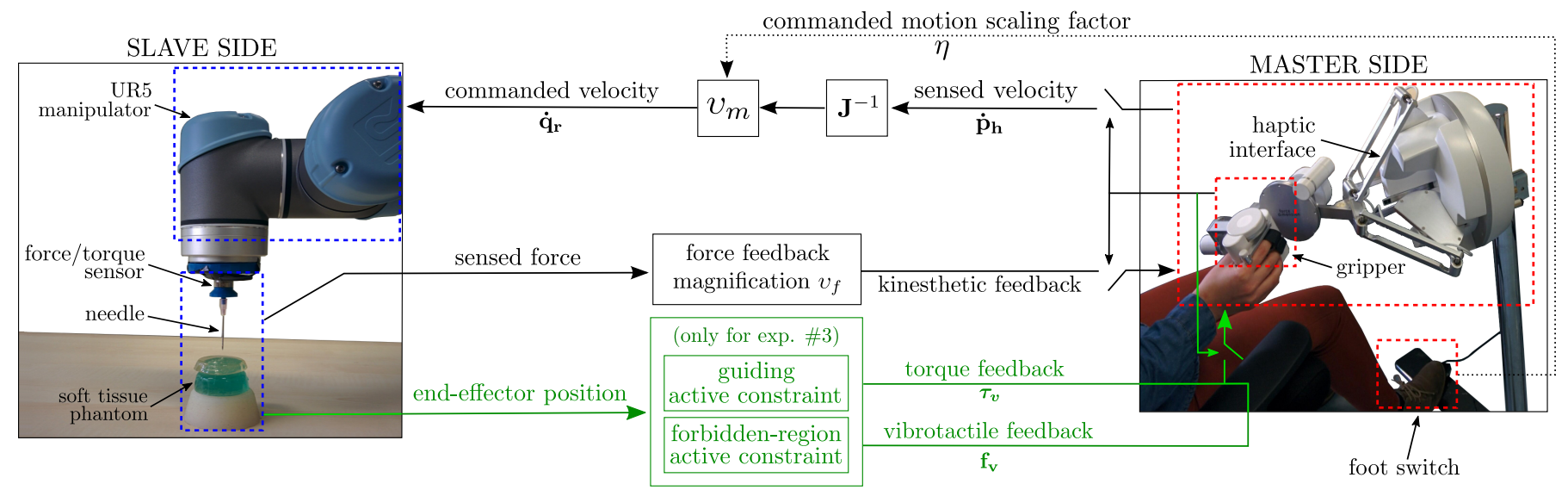

Fig. 2. Teleoperation system. The forces registered by the ATI force/torque sensor in the remote environment are provided to the human operator through the Sigma.7 haptic interface. The same interface is also used to control the motion of the UR5 robotic manipulator. For safety reasons, we used the gripper of the Sigma.7 to enable/disable the haptic feedback and the motion control of the slave robot. The master and slave systems are interconnected only when the gripper is closed. Finally, in the guidance experiment of Sec. II-B5, the human operator is also provided with virtual forces guiding him along a predetermined direction (guiding active constraint) and notifying him when the target depth of insertion is reached (forbidden-region active constraint).

states. Li et al. ${ }^{26}$ used granular jamming stiffness feedback actuators for simulating multi-fingered tissue palpation procedures in traditional and in robot-assisted minimally invasive surgery. Soft tissue stiffness is simulated by changing the stiffness property of the actuator during palpation. Gerovich et al. ${ }^{27}$ showed that haptic feedback, in comparison to not providing any force feedback, reduced puncture overshoot by at least $52 \%$ when visual feedback was absent or limited to static image overlay. More recently, Pacchierotti et al. ${ }^{28}$ presented a haptic-enabled teleoperation system for steering flexible needles. It enables clinicians to directly maneuver the surgical tool while providing them with navigation cues through kinesthetic and vibratory force feedback. Abayazid et al. ${ }^{4}$ extended this approach to co-manipulated needle insertion, in which the clinicians still maneuvered the surgical tool but an autonomous obstacle avoidance algorithm was enforced. In both cases, providing haptic feedback significantly improved the accuracy of the needle insertion task.

However, despite these expected clinical benefits, current commercially-available teleoperated surgical robots do not provide the surgeon with haptic feedback. This omission is mainly due to the negative effect that haptic force feedback may have on the stability and safety of the teleoperation system. Stability of such systems can be, in fact, significantly affected by communication latency in the teleoperation loop, hard contacts, relaxed grasps, and many other destabilizing factors which dramatically reduce the effectiveness of haptics in teleoperation, interfere with the surgery, and may be even dangerous for the patient. ${ }^{7,29,30}$

Stability issues become more critical when we aim at amplifying the force signals received at the master side. In fact, while providing magnified haptic feedback in teleoperation can lead to finer sensitivity and improved performance, ${ }^{11,31}$ it may severely affect the stability of the haptic loop. Magnified haptic feedback is commonly used in haptic-enabled microscale teleoperation, which requires scaling gains in the order of $10^{4}-10^{7}$, depending on the application. These high gains impose a rigid trade-off between stability and transparency.
Venture et al. ${ }^{32}$ addressed this problem by implementing a passivity-based position-position coupling scheme that ensures unconditional stability; and Kim et al. ${ }^{33}$ derived a relationship between performance, stability, and scaling factors of velocity (or position) and force. Experiments in different telemanipulation tasks, such as positioning, indenting, and nanolithography, showed the stability of both systems. Magnified haptic feedback can also be beneficial in robot-assisted surgery, enabling the operating surgeon to better discriminate different types of tissue during palpation or needle insertion, e.g., cancerous vs. healthy.

Given the expected benefits of haptic feedback and the challenges of stable implementation, many researchers have turned to sensory substitution techniques, wherein force information is presented via an alternative feedback channel, such as vibrotactile, ${ }^{34}$ auditory, ${ }^{35}$ or visual cues. ${ }^{36}$ Schoonmaker et al. ${ }^{34}$, for example, designed a vibrotactile feedback system to provide the surgeon with information about the force exerted on the surgical tools. Kitagawa et al. ${ }^{36}$ substituted direct haptic feedback with visual and auditory cues providing the surgeon with a representation of the forces applied at the slave side. $\mathrm{Cao}^{34}$ demonstrated that vibrotactile stimulation is a viable substitute for force feedback in minimally invasive surgery, enhancing surgeons' ability to control the forces applied to tissue and differentiate its softness in a simulated tissue probing task. Because no haptic forces are displayed to the operating surgeon, sensory substitution techniques make teleoperation systems intrinsically stable. ${ }^{14,19,37}$ However, although the stability of the system is guaranteed, the provided stimuli differ substantially from the ones being substituted (e.g., a beep sound instead of force feedback). Therefore, sensory substitution often shows performance inferior to that achieved with unaltered force feedback. 4,14,19,28

This paper presents the experimental evaluation of a hapticenabled teleoperation system for robot-assisted medical procedures. It addresses the safety challenges of providing magnified haptic feedback in three different scenarios: stiffness discrimination during palpation, stiffness discrimination dur- 
ing needle insertion, and guidance during needle insertion. Since haptic feedback technology is not yet commercially available, robotic minimally invasive systems are currently being used in procedures that can be completed without this information. However, for example, being able to effectively discern different types of tissue during surgery would enable the operating surgeon to feel exactly where tumors are located. Thanks to this specific knowledge, the surgery can be tailored to the patient's current disease state and all of the cancer can be removed while sparing as much of the patient's healthy tissue as possible. In this way, (magnified) haptics could enable surgeons to deliver better care in procedures they are already performing robotically, and it could also broaden the range of operations that can be done with a robotic surgical system. Similarly, current commercially-available systems for biopsy enable the clinician to manually insert the needle while being provided with visual feedback through ultrasound or CT systems. Being able to easily follow pre-determined trajectories during the insertion could enable clinicians to improve the accuracy of such delicate procedures. Inaccurate placement of the needle may in fact result in misdiagnosis and unsuccessful treatment during biopsy and brachytherapy, respectively. This work presents the first experimental evaluation of the effectiveness and safety of magnified haptic feedback for robot-assisted needle insertion and palpation, as well as the evaluation of the role of guiding and forbidden-region active constraints in such scenarios.

\section{Materials And MethodS}

\section{A. Robotic teleoperation system}

The proposed haptic-enabled teleoperation system is composed of a 7-DoF Sigma.7 haptic interface, a 6-DoF Universal Robot 5 manipulator, and an ATI Nano-17 six-axis force/torque sensor. The system enables the clinician to intuitively and accurately control the motion of the slave endeffector while providing him or her with compelling haptic feedback about the interaction with the remote environment. The integrated teleoperation system is shown in Figs. 1 and 2. The features of each device are reported in Table I.

a) Master haptic system: The master haptic system is composed of a 7-DoF Sigma.7 haptic interface. It is composed of a delta-based parallel kinematics structure that provides good closed-loop stiffness and high accuracy. The rotating wrist joints allow the user to also change the orientation of the gripper end-effector. Moreover, the interface is constructed in such a way that translations and rotations are decoupled from each other. All degrees of freedom are active, and it can provide up to $20 \mathrm{~N}$ in translation, $0.4 \mathrm{Nm}$ in rotation, and $8 \mathrm{~N}$ in grasping. This haptic interface is also equipped with active gravity compensation to improve the teleoperation transparency and reduce the clinician's fatigue. Finally, the system is equipped with a footswitch that we used to tune the motion scaling factor between master and slave.

b) Slave robotic system: The slave system is composed of an ATI Nano17 sensor mounted on the end-effector of a 6DoF manipulator Universal Robot 5. The Nano17 is a six-axis force/torque sensor with a diameter of $17 \mathrm{~mm}$ and a weight of
TABLE I

TELEOPERATION SYSTEM DETAILS AND PARAMETERS

\begin{tabular}{|c|c|}
\hline \multicolumn{2}{|l|}{ Master system } \\
\hline Hardware & Sigma.7 (Force Dimension, $\mathrm{CH}$ ) \\
\hline Refresh rate & $1 \mathrm{kHz}$ \\
\hline $\begin{array}{l}\text { Maximum forces } \\
\text { and torques }\end{array}$ & $\begin{array}{l}\text { translation } 20 \mathrm{~N} \text {, rotation } 400 \mathrm{mNm} \text {, } \\
\text { grasping } 8 \mathrm{~N}\end{array}$ \\
\hline \multicolumn{2}{|l|}{ Slave system } \\
\hline \multicolumn{2}{|l|}{ Robotic manipulator } \\
\hline Hardware & UR5 (Universal Robot, DK) \\
\hline Refresh rate & $125 \mathrm{~Hz}$ \\
\hline Speed & all joints $180 \mathrm{deg} / \mathrm{s}$, tool $1 \mathrm{~m} / \mathrm{s}$ \\
\hline \multicolumn{2}{|l|}{ Force/torque sensor } \\
\hline Hardware & Nano17 (ATI Industrial Automation, USA) \\
\hline Refresh rate & $1 \mathrm{kHz}$ \\
\hline Dimensions & $17 \times 14.5 \mathrm{~mm}($ diameter $\times$ height $)$ \\
\hline \multicolumn{2}{|l|}{ Interconnection } \\
\hline Hardware & real-time GNU/Linux machine \\
\hline Stability control & time-domain passivity control (see Fig. 3) \\
\hline $\begin{array}{l}\text { Motion scaling } \\
\text { factor }\end{array}$ & $0.1-0.3$ \\
\hline $\begin{array}{l}\text { Force scaling } \\
\text { factor }\end{array}$ & $5-15$ \\
\hline
\end{tabular}

$9 \mathrm{~g}$. It has high speed output, span temperature compensation, and high signal-to-noise ratio. The 6-DoF manipulator is able to move the end-effector at up to $1 \mathrm{~m} / \mathrm{s}$ and each joint at $180 \mathrm{deg} / \mathrm{s}$. Its repeatability is $\pm 0.1 \mathrm{~mm}$ for quick-precision handling with a maximum payload of $5 \mathrm{~kg}$. It is equipped with true absolute encoders to acquire absolute joints positions immediately upon power-up.

c) Implementation: The teleoperation system is managed by a GNU/Linux machine (Ubuntu 14.04.4 with Linux Kernel 4.2), equipped with a real-time scheduler.

In this work, we use the Sigma.7 as an impedance haptic interface. We measure the position of the master end-effector, controlled by the human operator, to set the position of the slave end-effector. At the same time, through the same master end-effector, we provide the operator with haptic force feedback from the remote environment.

The velocities of the robotic manipulator joints $\dot{\mathbf{q}} \in \mathbb{R}^{6}$ are commanded as

$$
\dot{\mathbf{q}}_{\mathbf{r}}=\mathbf{J}^{-1} \dot{\mathbf{p}}_{\mathbf{h}} v_{m},
$$

where $\mathbf{J}^{-1}$ is the inverse of the manipulator Jacobian matrix $\mathbf{J} \in \mathbb{R}^{6 \times 6},^{38} \dot{\mathbf{p}}_{\mathbf{h}} \in \mathbb{R}^{6}$ are the velocities of the haptic interface's end-effector, and $v_{m}$ is the scaling factor between the master and slave workspaces. Scaling factor $v_{m}=\zeta \eta$ is tunable in order to enable coarse and fine gestures at different stages of the operation. In this paper, $\zeta$ varies between 0.1 and 0.3 , according to the considered task (see Sec. II-B). On the other hand, $\eta$ is controlled by the footswitch: $\eta=1$ when 


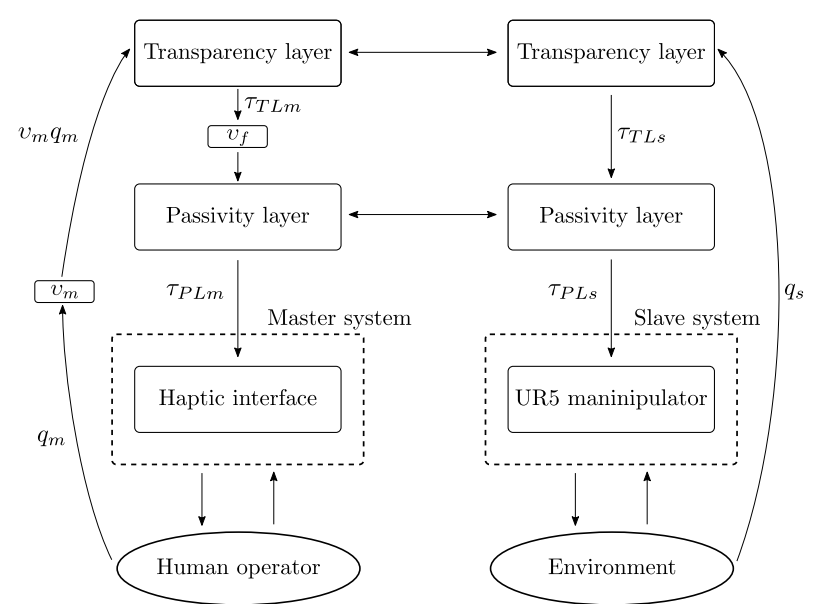

Fig. 3. Time-domain passivity controller. The Transparency Layer aims at achieving the desired transparency, while the Passivity Layer ensures the stability of the system.

the footswitch is not pressed and $\eta=3$ when the footswitch is pressed. This way of scaling the motion of the slave robot with respect to the master console has been inspired by the da Vinci Surgical System (Intuitive Surgical Inc., USA). In fact, in the da Vinci robot, the operating surgeon can switch between "normal" (master:slave, 2:1), "fine" (3:1, default), and "ultra-fine" (5:1) scaling factors by pressing the buttons on the left side pod of the master console.

The 7-DoF haptic interface registers the motion of the operator at $1 \mathrm{kHz}$, and it provides haptic feedback about the interaction of the slave end-effector with the remote environment at the same rate. The Universal Robot manipulator then follows the motion commanded by the haptic interface at $125 \mathrm{~Hz}$, according to eq. (1). Finally, the ATI force/torque sensor registers interaction forces at the slave side at $1 \mathrm{kHz}$. Finally, we used the gripper of the Sigma.7 haptic interface to enable/disable the motion control of the slave robot and the haptic feedback. When the gripper is closed, the motion of the slave robot is controlled by the master interface and the force sensed by the ATI sensor is provided to the operator through the same interface. Conversely, when the gripper is open, master and slave systems are disconnected (see Fig. 2). The operator does not receive any force feedback in this case, and he/she can move the end-effector of the master interface without affecting the position of the slave robot, e.g., to move it away from the edges of its workspace or to achieve a more comfortable orientation/position. For safety purposes, a small force of $0.5 \mathrm{~N}$ is always provided to the Sigma.7's gripper, to keep it open if no force is applied by the operator. The benefits of this control policy are twofold: (a) the workspace of the slave system is not limited either by the workspace of the haptic interface or by the motion scaling factor $v_{m}$; and (b) the slave robot moves only when there is an explicit will of the operator, improving the overall safety of the system. A video of the robotic teleoperation system controlled in free space is available as supplemental material and can be downloaded at http://goo.gl/oHe1y2.

d) Stability: Stability of teleoperation systems with force reflection can be significantly affected by communication

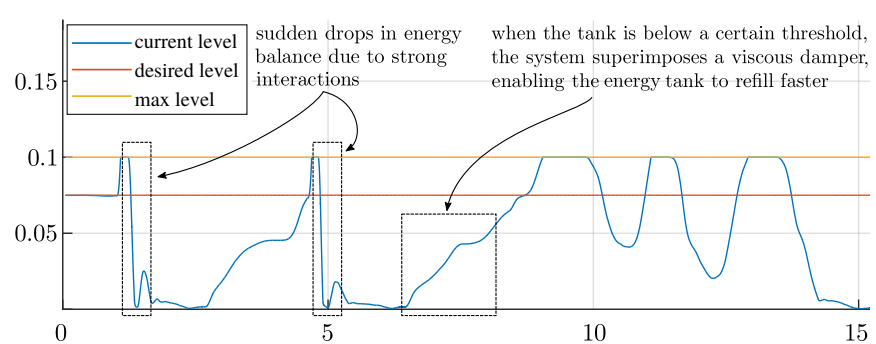

(a) tank level (J) vs. time (s)

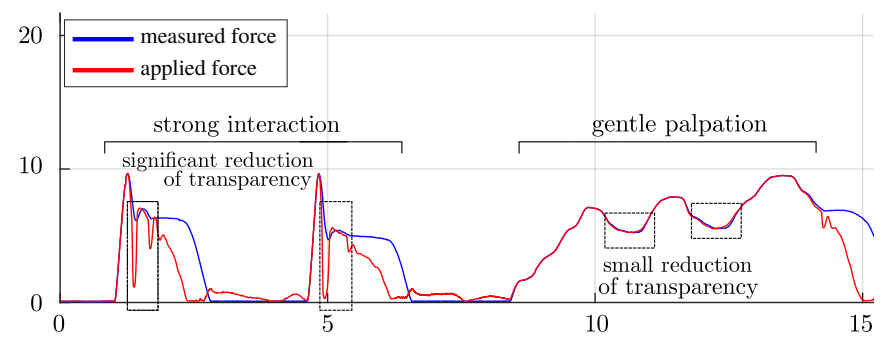

(b) force (N) vs. time (s)

Fig. 4. Representative run of a palpation task with the passivity controller enforced. (a) Maximum, desired, and current energy level of the tank in yellow, red, and blue, respectively. For the system to be passive, the energy has to be always greater than zero. Whenever the system risks to lose passivity, the Passivity Layer acts on the force feedback provided to the user, guaranteeing safety at the expense of transparency. (b) Ideal force computed by the Transparency Layer (blue, measured by the ATI sensor) and force feedback applied at the master side after the passivity check (red).

latencies, hard contacts, relaxed grasps, and many other timevarying destabilizing factors. Achieving stability is particularly challenging when amplifying the force fed back to the human operator. Instabilities in the teleoperation loop can lead to unwanted and possibly dangerous oscillations on both sides of the system, which can put in severe danger the safety of the patient and the operator. So as to preserve the stability (and safety) of the teleoperation system, we customized the timedomain passivity controller described $\mathrm{in}^{39}$. The architecture is split into two separate layers. The hierarchical top layer, named Transparency Layer, aims at achieving the desired transparency, while the lower layer, named Passivity Layer, ensures the passivity of the system (see Fig. 3).

The human operator and the environment impress a movement $q_{m}$ and $q_{s}$ to the master and slave systems, respectively. The Transparency Layer displays the desired behavior to obtain transparency by computing the torques $\tau_{T L m}$ and $\tau_{T L s}$ to be applied to the operator and the environment, respectively. $q_{m}$ and $\tau_{T L m}$ are modulated accordingly to the selected motion and force scaling factors, $v_{m}$ and $v_{f}$, respectively. The Passivity Layer then checks how the action planned by the Transparency Layer influences the energy balance of the system, whose level is saved into a virtual energy tank. For the system to be passive, the amount of energy in the tank has to be always greater than zero, i.e., the system should not provide more energy than that extracted from the environment and the operator. If the passivity condition is not violated, the planned action $\tau_{T L *}$ can be directly applied to both sides of the system. However, if loss of passivity is detected, a scaled control action $\tau_{P L *}$ is applied to preserve stability, resulting in a temporary loss of transparency. In general, in the same 
environmental conditions, a high force scaling factor $v_{f}$ will require the Passivity Layer to intervene more often than a low force scaling factor. For this reason, we expect the benefits of our force magnification approach to eventually hit a plateau, as the Passivity Layer will not allow the system to render forces that are too high. The level of this plateau is affected by various factors, such as communication latencies and the type of grasp.

Figure 4 shows an example of how our passivity controller works in a representative palpation trial. The operator is requested to apply first high and then low forces to a tissue phantom ("strong" and "gentle" palpation in Fig. 4). Fig. 4a shows the maximum, desired, and current energy level of the tank with yellow, red, and blue lines, respectively. In order to guarantee the passivity of the system, the energy level of the tank should always be greater than zero. Fig. 4b shows the magnitude of the forces measured on the slave side by the ATI sensor (blue) and the magnitude of the forces actually provided through the haptic interface on the master side (red). Whenever the force applied is different from the force measured, the Passivity Layer has intervened to guarantee the stability of the teleoperation system, at the expense of its transparency. As it is clear by comparing strong vs. gentle interaction phases, when there are force peaks the Passivity Layer needs to take action to guarantee the safety of the system. Finally, Fig. 4 also shows that the controller can actively extract energy from the operator when necessary: whenever the tank level is below a certain desired threshold (red in Fig. 4a), the system superimposes a viscous damper to the force feedback applied to the operator, making the energy tank refill faster. As mentioned in Sec. I, preserving the stability of the system is of paramount importance. This task is particularly challenging when dealing with magnified haptic feedback (see Sec. II-B). A video showing the system behavior with and without enforcing the passivity control technique is available as supplemental material and can be downloaded at http://goo.gl/pYRWqS. Data in Fig. 4 is taken from the trial shown in the video (when the passivity controller is enabled).

\section{B. Experimental evaluation}

In order to demonstrate the effectiveness of magnified haptic feedback in such medical procedures, we conducted three experiments.

The first experiment evaluates the proposed system in a stiffness discrimination task during tool-mediated palpation of soft tissue; the second experiment evaluates the proposed system in a stiffness discrimination task during needle insertion in soft tissue; and the third experiment evaluates our system in following a predetermined direction when inserting a needle in soft tissue. Before running these experiments, we also characterized the mechanical properties of the soft tissue phantoms being used.

1) Characterization of soft tissue phantoms: Ex vivo tissue has been used in research to mimic the mechanical properties of living tissue. Clinician trainees often use turkey breast to mimic human breast in needle insertion training. ${ }^{40} \mathrm{Pig}$ liver $^{41}$ and bovine liver ${ }^{42}$ are other popular choices for medical

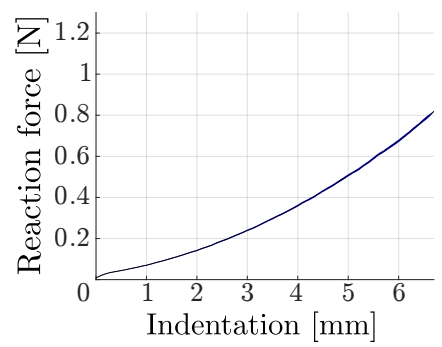

(a) $7 \%$ - mat1

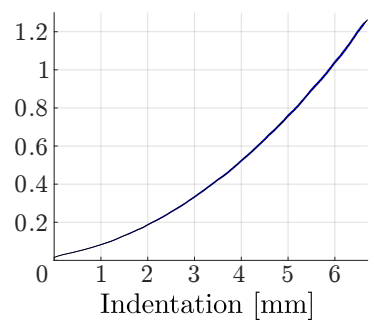

(b) $7.25 \%-$ mat 2
Fig. 5. Mean force recorded along the z-axis (see Fig. 1) during indentation experiments. The test was performed on tissue phantoms prepared with mixtures of $7 \%$ (mat 1 ) and $7.25 \%$ (mat 2 ) of bovine gelatin powder and water. Blue patches indicate the standard deviation of the mean force during each indentation experiment.

training. Unfortunately, these types of tissue have structures which make experiments not very repeatable and may even contain harmful bacteria. ${ }^{43}$ For this reason, researchers often use artificial tissues, which can be made translucent, homogeneous, and are generally safer to handle than ex vivo tissue. A popular choice is the rubber-like material Polyvinyl chloride (PVC), which lasts years with slight changes in its mechanical characteristics. ${ }^{44}$ Organic artificial tissues, such as gelatin from pig skin and beef bones, are also widely used as human tissue mimics during needle insertion and palpation tasks. ${ }^{45}$ They are safer to handle than ex vivo tissues and they are easy to retrieve. For these reasons, in our work we used bovine gelatin produced by Sleaford Quality Foods Ltd. The samples were allowed to reach room temperature prior to experimentation. Their behavior did not noticeably change throughout the experiments.

We carried out two experiments to characterize the soft tissue phantoms used during the experimental evaluations of Secs. II-B3, II-B4, and II-B5, inspired by the work presented by Wedlick and Okamura. ${ }^{46}$ In the first experiment, we attached a spherical-ended conical indenter to the ATI sensor, which was in turn fixed to the end-effector of the robotic manipulator (end-effector "A" in Fig. 1). The indenter has a diameter of $2 \mathrm{~mm}$ at the tip and a length of $55 \mathrm{~mm}$. Interaction forces were recorded while the tool was indented into the tissue at $2 \mathrm{~mm} / \mathrm{s}$, for a total displacement of $8 \mathrm{~mm}$, and then retracted. ${ }^{46}$ The indentation did not break the tissue phantom. Each indentation trial was repeated 5 times. To match the phantoms used in Sec. II-B3, the indentation test was performed on tissue phantoms prepared with mixtures of $7 \%$ and $7.25 \%$ of bovine gelatin powder and water, referred to as mat1 and mat2, respectively. Fig. 5 shows the interaction forces vs. indentation recorded during this experiment. The difference in stiffness between the two considered materials is smaller than the difference in stiffness in cancerous vs. healthy tissues. ${ }^{47,48}$ Only forces along the $z$-axis (see Fig. 1) were considered.

In the second experiment, we attached a 18-gauge prostate brachytherapy seeding needle (Worldwide Medical Technologies, LLC) to the ATI sensor, which was again fixed to the end-effector of the robotic manipulator (end-effector " $\mathrm{B}$ " in Fig. 1). First, the needle was inserted for $2.25 \mathrm{~cm}$, until the 


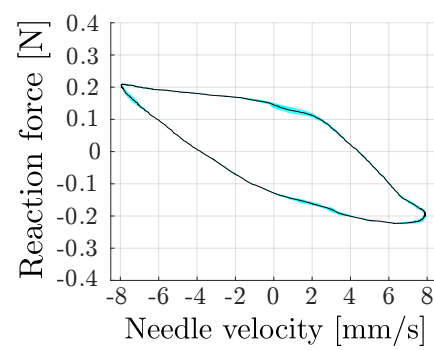

(a) $7 \%-$ mat 1

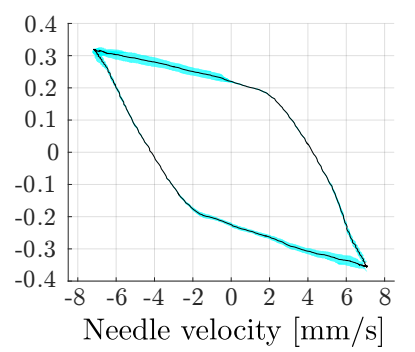

(b) $7.75 \%$ - mat3
Fig. 6. Mean force recorded between the needle and the soft tissue during insertion experiments. The test was performed on tissue phantoms prepared with mixtures of $7 \%$ (mat 1 ) and $7.75 \%$ (mat 3 ) of bovine gelatin powder and water. Cyan patches indicate the standard deviation of the mean force during each insertion experiment.

needle tip extended $1.25 \mathrm{~cm}$ beyond the tissue. The needle was then held stationary for 60 seconds, so that the tissue could relax. Finally, the needle was cyclically retracted and inserted with a $1 \mathrm{~cm}$ peak-to-peak amplitude. Experiments were conducted using sinusoidal motion with $3 \mathrm{~s}$ period. ${ }^{46}$ Each cycle (retraction + insertion) was repeated 5 times. To match the phantoms used in Secs. II-B4 and II-B5, this experiment was performed on tissue phantoms prepared with mixtures of $7 \%$ and $7.75 \%$ of bovine gelatin powder and water, referred to as mat 1 and mat3, respectively. Fig. 6 shows the interaction forces vs. needle velocity for this experiment.

2) Participants: Thirteen right-handed subjects (nine males, four females, average age 29) participated in the study presented in the following sections. Five of them had previous experience with haptic interfaces. None of the participants reported any deficiencies in their visual or haptic perception abilities. Participants were briefed about all the tasks and afterwards signed an informed consent, including the declaration of having no conflict of interest. All of them were able to give the consent autonomously. The participation in the experiment did not involve the processing of genetic information or personal data (e.g., health, sexual, lifestyle, ethnicity, political opinion, religious or philosophical conviction). Our organization does not require any IRB review for this case.

3) Experiment \#1: stiffness discrimination - palpation: The first experiment evaluates the proposed teleoperation system in a stiffness discrimination task during tool-mediated palpation of soft tissue. Participants were asked to interact with two different soft tissue phantoms and indicate which one was the stiffest. We used phantoms prepared with mixtures of $7 \%$ and $7.25 \%$ of bovine gelatin powder and water, which we referred to as matl and mat2 (see Sec. II-B1).

a) Methods: We carried out this palpation experiment in two different modalities: direct hand interaction $(\mathrm{H})$ and robotic teleoperation $(\mathrm{R})$. A video of the experiment in teleoperation is available as supplemental material and can be downloaded at http://goo.gl/WUHWFb.

During direct hand interaction $(\mathrm{H})$, subjects were blindfolded and required to interact with the two soft tissue phantoms by means of the conical indenter " $\mathrm{A}$ " shown in Fig. 1, detached from the robot. The same indenter was used in Sec. II-B1 to characterize the elastic properties of the two materials. Subjects were asked to hold the upper extremity of the indenter with their right thumb and index fingers, touch the two phantoms for as long as they wanted, and tell the experimenter which one felt the stiffest.

During teleoperated interaction (R), subjects were blindfolded and required to interact with the two soft tissue phantoms through the proposed haptic-enabled teleoperation system. The same conical indenter used above was attached to the end-effector of the slave robot, as shown in Fig. 1. Subjects controlled the motion of the slave robot through the Sigma.7 haptic interface, and the forces sensed by the ATI sensor were provided to the subjects through the same haptic interface, as detailed in Sec. II-A. The master-slave motion scaling factor $\zeta$ was set to 0.1 (see eq. (1)), i.e., moving the end-effector of the Sigma interface of $10 \mathrm{~cm}$ moves the indenter of $1 \mathrm{~cm}$. The contact forces due to the interaction between the slave robot and the phantoms were magnified and provided to the subjects through the Sigma.7 haptic interface. We considered three magnification rates: $5 \times, 10 \times$, and $15 \times$. Subjects were again asked to touch the two phantoms for as long as they wanted and tell the experimenter which one felt the stiffest.

We carried out 80 repetitions of the palpation task, 20 for each feedback condition: direct hand interaction $(\mathrm{H})$, teleoperation with magnification $5 \times(\mathrm{R} 5 \times)$, teleoperation with magnification $10 \times(\mathrm{R} 10 \times)$, and teleoperation with magnification $15 \times(\mathrm{R} 15 \times)$. After each repetition, the relative position of the two phantoms was randomly changed. Results are reported in Sec. III-A.

4) Experiment \#2: stiffness discrimination - needle insertion: The second experiment evaluates the proposed teleoperation system in a stiffness discrimination task during needle insertion in soft tissue. Participants were asked to insert a 18gauge prostate brachytherapy needle inside two different soft tissue phantoms piled up, and stop the insertion as soon as they felt the change in stiffness. We used phantoms prepared with mixtures of $7 \%$ and $7.75 \%$ of bovine gelatin powder and water, which we referred to as mat 1 and mat3 (see Sec. II-B1).

a) Methods: Two soft tissue phantoms were placed one on top of the other, with the stiffer phantom placed below the softer one. Similarly to the palpation experiment, we carried out this needle insertion task in two different modalities: direct hand interaction $(\mathrm{H})$ and robotic teleoperation $(\mathrm{R})$. A video of the experiment in teleoperation is available as supplemental material and can be downloaded at http://goo.gl/QWfqlI.

During direct hand interaction $(\mathrm{H})$, subjects were blindfolded and required to insert the 18-gauge needle in the soft tissue phantoms using their hands. Subjects were asked to hold the hub of needle with their right thumb and index fingers, insert the needle perpendicularly to the tissue surface, and stop the insertion as soon as they felt penetrating inside the lower (stiffer) phantom.

During teleoperated interaction $(\mathrm{R})$, subjects were blindfolded and required to insert the needle using the proposed haptic-enabled teleoperation system. They were again asked to insert the needle perpendicularly to the tissue surface, and stop the insertion as soon as they felt penetrating inside the lower (stiffer) phantom. The same 18-gauge needle used above was attached to the end-effector of the slave robot, as shown 
in Fig. 1 (end-effector "B"). Similarly to Sec. II-B3, subjects controlled the motion of the slave robot through the Sigma.7 haptic interface, and the forces sensed by the ATI sensor were provided to the subjects through the same haptic interface (see Sec. II-A). The master-slave motion scaling factor $\zeta$ was set to 0.3 (see eq. (1)), i.e., moving the end-effector of the Sigma interface of $3 \mathrm{~cm}$ moves the indenter of $1 \mathrm{~cm}$. The contact forces due to the interaction between the slave robot and the phantoms were magnified and provided to the subjects through the Sigma.7 haptic interface. Similarly to Sec. II-B3, we considered three magnification rates: $5 \times, 10 \times$, and $15 \times$.

We carried out 80 repetitions of the needle insertion task, 20 for each feedback condition: direct hand interaction $(\mathrm{H})$, teleoperation with magnification $5 \times(\mathrm{R} 5 \times)$, teleoperation with magnification $10 \times(\mathrm{R} 10 \times)$, and teleoperation with magnification $15 \times(\mathrm{R} 15 \times)$. After each repetition, we took a highresolution picture of the environment to measure the depth of insertion of the needle inside the tissue phantoms. Results are reported in Sec. III-B.

5) Experiment \#3: needle insertion along a predetermined direction: Active constraints are software functions used in assistive robotic systems to regulate the motion of surgical tools. The motion of the surgical tool, the needle in our case, is still controlled by the surgeon, but the system constantly monitors its motion and takes some actions if it fails to follow a predetermined procedure. Active constraints play two main roles: they can either guide the motion of the tool or strictly forbid the surgeon from reaching certain regions. ${ }^{49} \mathrm{~A}$ guiding active constraint attenuates the motion of the surgical tool in some predefined directions to encourage the surgeon to conform to the procedure plan. A forbidden-region active constraint seeks to prevent the needle from entering a specific region of the workspace. Forbidden-region active constraints may be introduced to protect areas that must be avoided to prevent damage of tissue. This is the case, for instance, of brain surgery, in which tissue manipulation in certain areas can cause serious injury to patients.

This last experiment aims at evaluating the effectiveness of our system in providing the clinician with haptic guidance during needle insertion in soft tissue. In particular, the task consisted in inserting a 18-gauge prostate brachytherapy needle in soft tissue while maintaining a predetermined inclination. Moreover, subjects were also required to stop the insertion of the needle when it reached a predetermined target depth. We used a guiding active constraint to indicate the desired needle inclination and a forbidden-region active constraint to indicate when to stop the insertion. Although active constraints have been used in different medical procedures, ${ }^{49}$ this is the first implementation of active constraints together with magnified haptic feedback. The clinician is provided, at the same time, with haptic information about the mechanical properties of the tissue being penetrated, guidance on the desired inclination of insertion, and an alert when the target depth has been reached.

a) Guiding active constraint: To maintain the correct inclination of the needle during the insertion, we provide torque feedback through the end-effector of the Sigma.7 haptic interface. The difference between the desired needle orientation $\mathbf{R}_{\mathbf{d}} \in \mathbb{R}^{3 \times 3}$ and the current needle orientation

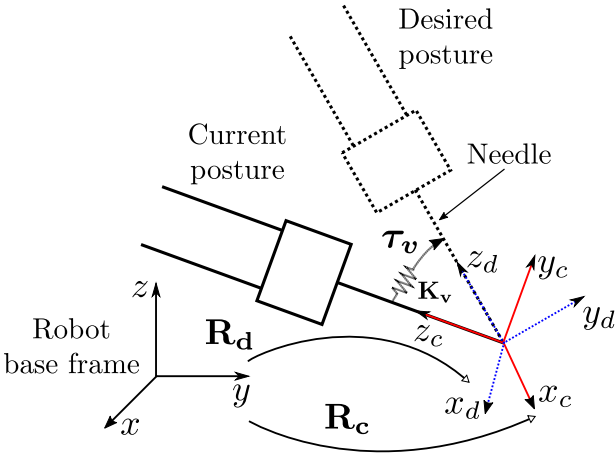

Fig. 7. Needle posture adjustment by using torque feedback. $\tau_{v}$ is the virtual torque to apply to the operator's hand. It is computed from the difference between the current and the desired postures, $\mathbf{R}_{\mathbf{c}}$ and $\mathbf{R}_{\mathbf{d}}$ respectively, through the virtual torsional stiffness $\mathbf{K}_{\mathbf{v}}$.

$\mathbf{R}_{\mathbf{c}} \in \mathbb{R}^{3 \times 3}$ w.r.t the robot's base generates a torque feedback $\boldsymbol{\tau}_{\boldsymbol{v}} \in \mathbb{R}^{3 \times 1}$ that gently keep the wrist of the Sigma.7 at the desired orientation (see Fig. 7). This orientation difference can be expressed as

$$
\mathbf{R}_{\mathrm{cd}}=\mathbf{R}_{\mathrm{c}}^{\mathbf{T}} \mathbf{R}_{\mathrm{d}}
$$

Torque feedback $\tau_{v}$ w.r.t. the robot base frame is then evaluated as

$$
\tau_{v}=\mathbf{K}_{\mathbf{v}} \Psi\left(\mathbf{R}_{\mathbf{c d}}\right),
$$

where $\Psi$ denotes a function that computes the three Euler angles from a rotation matrix, ${ }^{50}$

$$
\mathbf{K}_{\mathbf{v}}=\left[\begin{array}{ccc}
k_{x} & 0 & 0 \\
0 & k_{y} & 0 \\
0 & 0 & k_{z}
\end{array}\right],
$$

is the torsional elastic constant matrix, and $k_{x}=k_{y}=k_{z}=$ $0.5 \mathrm{Nm} / \mathrm{rad}$ are the torsional elastic constants for the three axes. Since the Sigma.7 haptic interface controls the motion of the slave robot, adjusting the orientation of the Sigma.7 also adjusts the orientation of the needle in the operating environment (see Sec. II-A). The virtual forces generated by the guiding active constraints are considered by the passivity controller and properly modulated to guarantee the stability of the system.

b) Forbidden-region active constraint: To notify the clinician when the target insertion depth is reached, we provide vibrotactile feedback through the end-effector of the Sigma.7 interface. Providing different types of haptic stimuli (i.e., kinesthetic and vibrotactile) to provide different types of information (i.e., orientation and forbidden regions) has already been proven to be a very effective approach in the teleoperation of surgical tools. ${ }^{51,52}$ In fact, it enables the clinician to easily discriminate between multiple pieces of information provided through the same perceptual channel.

As soon as the needle reaches the target depth of incision, the clinician is notified through a 200 -ms-long vibration burst. 


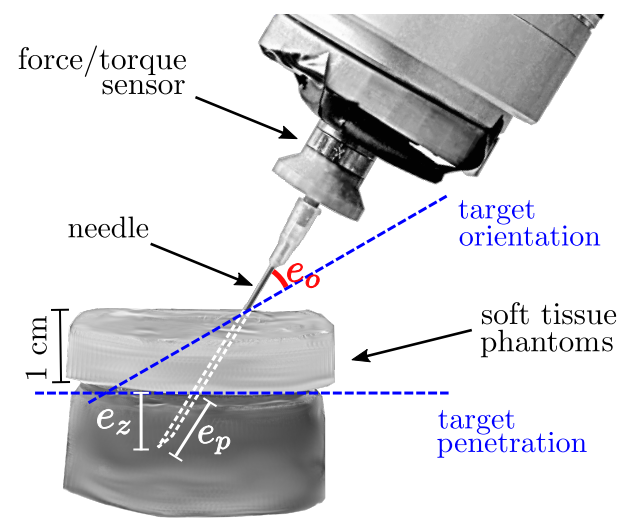

Fig. 8. Experiment \#3: needle insertion along a predetermined direction. A guiding active-constraint gently guides the operator to keep the desired needle orientation, while a forbidden-region active constraint notifies the operator when the target insertion depth is reached. We evaluated the error in keeping the desired needle orientation $e_{o}$, the error in achieving the desired penetration depth $e_{p}$, and its projection on the z-axis $e_{z}$.

This force $\mathbf{f}_{\mathbf{v}} \in \mathbb{R}^{3 \times 1}$, provided by the Sigma.7, is

$$
\mathbf{f}_{\mathbf{v}}(t)=\left[\begin{array}{c}
0 \\
0 \\
A \operatorname{sgn}(\sin (\pi f t))
\end{array}\right],
$$

where $A=1 \mathrm{~N}$ and $f=200 \mathrm{~Hz}$ are the amplitude and frequency of the vibration, respectively. These values were chosen to maximally stimulate the Pacinian corpuscle receptors, ${ }^{53}$ be easy to distinguish, ${ }^{54}$ and fit the master device specifications. Although the vibration was rendered only along the $z$-direction (see Fig. 1), users were not able to perceive the directional information. ${ }^{55} \mathrm{~A}$ notch filter prevented the vibrations applied at the master side to affect the motion of the slave robot. The virtual forces generated by the forbiddenregion active constraint are not modulated by the passivity controller, since vibrotactile feedback does not affect the stability of teleoperation systems. ${ }^{21}$

c) Methods: Similarly to the previous experiment, two soft tissue phantoms were placed one on top of the other. However, this time, both phantoms had the same stiffness. They were both prepared with mixture of $7 \%$ of bovine gelatin powder and water, referred to as matl (see Sec. II-B1). The top phantom was $1 \mathrm{~cm}$ thick, while the bottom phantom was $3 \mathrm{~cm}$ thick (see Fig. 8). We also added blue dye in the mixture of the bottom phantom. We carried out this needle insertion experiment in two different modalities: direct hand interaction $(\mathrm{H})$ and robotic teleoperation $(\mathrm{R})$. Subjects were required to perform insertions at three predetermined inclinations with respect to the surface of the phantom: $30^{\circ}$, $50^{\circ}$, and $70^{\circ}$. A video of the experiment in teleoperation is available as supplemental material and can be downloaded at http://goo.gl/EdBtO1.

During direct hand interaction $(\mathrm{H})$, subjects were required to insert the 18-gauge needle in the soft tissue phantom using their hands. They were asked to hold the hub of the needle with their right thumb and index fingers, insert the needle in the phantom with the target inclination, and stop the insertion as soon as the needle reached the surface of the second (blue) phantom. This time subjects were able to see the environment and the needle.

During teleoperated interaction (R), subjects were required to insert the 18-gauge needle in the soft tissue phantom using the proposed haptic-enabled teleoperation system. The same 18-gauge needle used above was attached to the end-effector of the slave robot, as shown in Fig. 1 (end-effector "B"). Similarly to Secs. II-B3 and II-B4, subjects controlled the motion of the slave robot through the Sigma.7 haptic interface, and the forces sensed by the ATI sensor were provided to the subjects through the same haptic interface (see Sec. II-A) with a fixed magnification factor of $10 \times$. Although subjects were not asked to directly use the force information from the ATI sensor - no discrimination of stiffnesses was requested here -, all the stability-related problems due to the force magnification were still present, aggravated by the additional virtual forces generated by the guiding active constraints. The master-slave motion scaling factor $\zeta$ was set to 0.3 (see eq. (1)), i.e., moving the end-effector of the Sigma interface of $3 \mathrm{~cm}$ moves the indenter of $1 \mathrm{~cm}$.

With respect to more simple fixed-direction needle guiding systems, the proposed approach has the great advantage of providing haptic feedback about the mechanical properties of the tissue being penetrated. Moreover, although clinicians can easily feel the haptic information provided by the two active constraints, they can still decide to not comply with the suggested insertion plan and act differently. In this way, at the same time, we can benefit from the precision of robotic guidance systems and the experience of skilled clinicians. Finally, this guidance system is quite flexible. It would be, in fact, quite easy to extend its capabilities to also drive flexible thin needle, as we have preliminarily demonstrated in ${ }^{4,28}$.

We carried out 24 repetitions of the needle insertion task, 4 for each target needle orientation $\left(30^{\circ}, 50^{\circ}, 70^{\circ}\right)$ and experimental condition (direct hand interaction and robotic teleoperation). After each repetition, we took a high-resolution picture of the environment to measure the penetration depth and the shaft inclination with respect to the surface of the soft tissue phantoms. Results are reported in Sec. III-C.

\section{RESUlTS}

This section reports the results and statistical analysis of the experiments reported in Secs. II-B3, II-B4, and II-B5.

\section{A. Experiment \#1: stiffness discrimination - palpation}

Fig. 9a shows the discrimination results in the four experimental conditions. The collected data passed the ShapiroWilk normality test and Mauchly's Test of Sphericity. A repeated-measures ANOVA showed a statistically significant difference between the means of the four feedback conditions $\left(\mathrm{F}_{3,36}=32.548, p<0.001, \mathrm{a}=0.05\right)$. Post-hoc analysis (Games-Howell post-hoc test) revealed statistically significant differences between all conditions but R10× vs. R $15 \times$. For this and all the following sets of data, statistically different $p$ values are reported in the corresponding figure. 


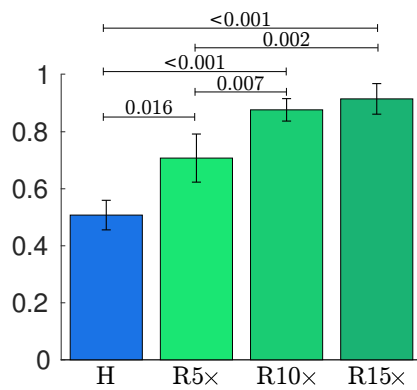

(a) Recognition Rate.

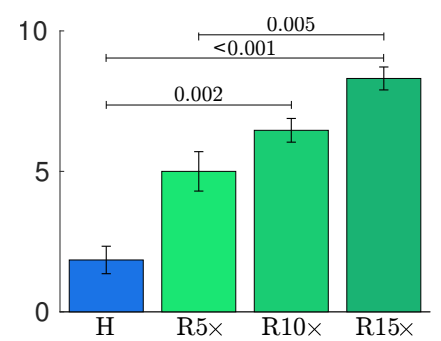

(b) Perceived Effectiveness.
Fig. 9. Experiment \#1: stiffness discrimination - palpation. Mean and 95\% confidence interval of (a) stiffness recognition results and (b) perceived effectiveness of the feedback conditions are plotted, during direct hand interaction $(\mathrm{H})$ and robotic teleoperation $(\mathrm{R})$ at different force magnification factors $(5 \times, 10 \times$, and $15 \times)$. In (a) correct answers are normalized on the number of trials proposed, while in (b) the rating given by the subject could be in the range 1-10. Higher values indicate better performance in accomplishing the given task.

In addition to the quantitative evaluation reported above, we also measured users' experience. Immediately after the experiment, subjects were asked to report the effectiveness of each feedback condition in completing the given task using bipolar Likert-type nine-point scales. Fig. 9b shows the perceived effectiveness for the four experimental conditions. A Friedman test showed a statistically significant difference between the means of the four feedback conditions $\left(\chi^{2}(3)=\right.$ $36.240, p<0.001, a=0.05)$. The Friedman test is the nonparametric equivalent of the more popular repeated-measures ANOVA. The latter is not appropriate here since the dependent variable was measured at the ordinal level. Post hoc analysis with Bonferroni adjustments revealed a statistically significant difference between $\mathrm{H}$ vs. $\mathrm{R} 10 \times, \mathrm{H}$ vs. $\mathrm{R} 15 \times$, and $\mathrm{R} 5 \times$ vs. $\mathrm{R} 15 \times$, while H vs. $\mathrm{R} 5 \times$ fell short of significance $(p=0.09)$. The Bonferroni correction is used to reduce the chances of obtaining false-positive results when multiple pair-wise tests are performed on a single set of data.

Finally, all thirteen subjects found conditions employing the teleoperation system to be the most effective at letting them detect the difference in stiffness between the considered materials.

Experimental conditions, task, and results of this experiment are summarized in Tab. II.

\section{B. Experiment \#2: stiffness discrimination - needle insertion}

Fig. 10a shows the penetration error in the four experimental conditions, evaluated as the portion of the needle penetrated inside the lower (stiffer) phantom. The collected data passed the Shapiro-Wilk normality test. Mauchly's Test of Sphericity indicated that the assumption of sphericity had been violated $\left(\chi^{2}(5)=13.738, p=0.018\right)$. A repeated-measures ANOVA with a Greenhouse-Geisser correction showed a statistically significant difference between the means of the four feedback conditions $\left(F_{1.98,23.80}=32.763, p<0.001, a=0.05\right)$. Posthoc analysis (Games-Howell post-hoc test) revealed statistically significant differences between all conditions.

Similarly as before, immediately after the experiment, subjects were asked to report the effectiveness of each feedback
TABLE II

SUMMARY OF EXPERIMENT \#1: STIFFNESS DISCRIMINATION - PALPATION

\begin{tabular}{|c|c|c|}
\hline Subjects & \multicolumn{2}{|l|}{13 (9 males, 4 females) } \\
\hline Task & \multicolumn{2}{|c|}{$\begin{array}{l}\text { Interacting with two different soft tissue phantoms and tell the } \\
\text { experiment which one felt stiffer }\end{array}$} \\
\hline Conditions & \multicolumn{2}{|l|}{ Direct hand interaction $(\mathrm{H})$} \\
\hline & \multicolumn{2}{|c|}{ Robotic teleoperation at a force magnification factor of $5(\mathrm{R} 5 \times)$} \\
\hline & \multicolumn{2}{|c|}{ Robotic teleoperation at a force magnification factor of $10(\mathrm{R} 10 \times)$} \\
\hline & \multicolumn{2}{|c|}{ Robotic teleoperation at a force magnification factor of $15(\mathrm{R} 15 \times)$} \\
\hline \multicolumn{3}{|c|}{ Motion scaling factor $\zeta \quad 0.1$} \\
\hline \multicolumn{3}{|c|}{ Best conditions (average \pm standard deviation) } \\
\hline \multicolumn{2}{|c|}{ Recognition rate ( $0-1$ range) } & $\mathrm{R} 15 \times: 0.9 \pm 0.1$ \\
\hline \multicolumn{2}{|c|}{ Perceived effectiveness (1-10 range) } & $\mathrm{R} 15 \times: 8.3 \pm 0.7$ \\
\hline \multicolumn{3}{|c|}{ Worst conditions (average \pm standard deviation) } \\
\hline \multicolumn{2}{|c|}{ Recognition rate ( $0-1$ range) } & $\mathrm{H}: 0.5 \pm 0.1$ \\
\hline \multicolumn{3}{|c|}{ Perceived effectiveness (1-10 range) } \\
\hline \multicolumn{3}{|c|}{ Statistical analysis (repeated-measures ANOVA, $a=0.05$ ) } \\
\hline \multicolumn{3}{|c|}{ Recognition rate (significant $p$ values only) } \\
\hline \multicolumn{2}{|r|}{ H vs. $R 5 \times$} & $p=0.016$ \\
\hline \multicolumn{2}{|r|}{ H vs. $R 10 \times$} & $p<0.001$ \\
\hline \multicolumn{2}{|r|}{ H vs. $\mathrm{R} 15 \times$} & $p<0.001$ \\
\hline \multicolumn{2}{|r|}{ R $5 \times$ vs. $\mathrm{R} 10 \times$} & $p=0.007$ \\
\hline \multicolumn{2}{|r|}{ R $5 \times$ vs. R $15 \times$} & $p=0.002$ \\
\hline \multicolumn{3}{|c|}{ Statistical analysis (related-samples Wilcoxon signed rank, $a=0.05$ ) } \\
\hline \multicolumn{3}{|c|}{ Perceived effectiveness (significant $p$ values only) } \\
\hline \multicolumn{2}{|r|}{ H vs. $R 10 \times$} & $p=0.002$ \\
\hline \multicolumn{2}{|r|}{ H vs. R15× } & $p<0.001$ \\
\hline \multicolumn{2}{|r|}{ R5× vs. R15× } & $p=0.005$ \\
\hline
\end{tabular}

condition in completing the given task using bipolar Likerttype nine-point scales. Fig. 10b shows the perceived effectiveness for the four experimental conditions. A Friedman test showed a statistically significant difference between the means of the four feedback conditions $\left(\chi^{2}(3)=38.721, p<\right.$ $0.001, a=0.05)$. Post hoc analysis with Bonferroni adjustments revealed a statistically significant difference between $\mathrm{H}$ vs. R5×, H vs. R10×, H vs. R15×, and R5× vs. R15×.

Again, all thirteen subjects found conditions employing the teleoperation system to be the most effective at letting them detect the difference in stiffness between the considered materials.

Experimental conditions, task, and results of this experiment are summarized in Tab. III.

\section{Experiment \#3: needle insertion along a predetermined direction}

As a measure of performance, we evaluated (1) the error in keeping the desired needle orientation $e_{o}$, (2) the error in achieving the desired penetration depth $e_{p}$, and (3) its projection on the normal direction $e_{z}$ (see Fig. 8). To compare the different metrics, we ran two-way repeated-measures 


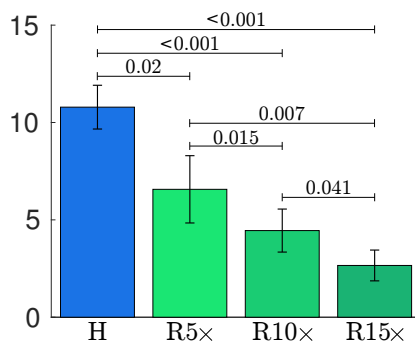

(a) Penetration error $(\mathrm{mm})$.

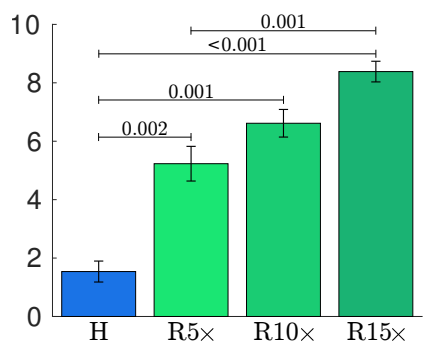

(b) Perceived Effectiveness.
Fig. 10. Experiment \#2: stiffness discrimination - needle insertion. Mean and 95\% confidence interval of (a) the penetration error and of (b) the perceived effectiveness are plotted when the task was performed by hand $(\mathrm{H})$ or by using the robot $(\mathrm{R})$, with force magnification factors $5 \times, 10 \times$, and $15 \times$. In (a), lower values indicate better performance in accomplishing the given task, while in (b), higher values correspond to a greater preference expressed by the subjects.

ANOVAs. Experimental setup (hand vs. teleoperation system) and insertion angle $\left(30^{\circ}, 50^{\circ}, 70^{\circ}\right)$ were treated as withinsubject factors.

Fig. 11a shows the insertion orientation error $e_{o}$ for the six experimental conditions, calculated as the absolute difference between the target orientation $\left(30^{\circ}, 50^{\circ}\right.$, or $\left.70^{\circ}\right)$ and the orientation of the needle at the end of the insertion task (see Fig. 8). All the data passed the Shapiro-Wilk normality test and the Mauchly's Test of Sphericity. Sphericity was assumed for variables with only two levels of repeated measures. The two-way repeated-measure ANOVA revealed a statistically significant difference only for the experimental setup $\left(F_{1,12}=\right.$ 46.844, $p<0.001, a=0.05$ ).

Fig. 11b shows the insertion penetration error $e_{p}$ for the six experimental conditions, calculated as the penetration of the needle inside the blue gelatin (see Fig. 8). All the data passed the Shapiro-Wilk normality test and the Mauchly's Test of Sphericity. Sphericity was assumed for variables with only two levels of repeated measures. The two-way repeated-measure ANOVA revealed again a statistically significant change only in the experimental setup $\left(F_{1,12}=66.255, p<0.001, a=\right.$ 0.05).

Fig. 11c shows the insertion penetration error along the $z$ direction $e_{z}$ (see Fig. 8). All the data passed the ShapiroWilk normality test and the Mauchly's Test of Sphericity. Sphericity was assumed for variables with only two levels of repeated measures. The two-way repeated-measure ANOVA revealed a statistically significant two-way interaction between experimental setup and insertion angle $\left(F_{2,22}=3.610, p<\right.$ $0.043, a=0.05)$. Interpreting the simple main effects ${ }^{56}$ for the experimental setup variable, we found a statistically significant difference between all the pairs with the same insertion angle: $\mathrm{H} 30^{\circ}$ vs. R30 ${ }^{\circ}, F_{1,12}=64.231, p<0.001$; $\mathrm{H} 50^{\circ}$ vs. $\mathrm{R} 50^{\circ}, F_{1,12}=23.779, p<0.001$; and $\mathrm{H} 70^{\circ}$ vs. $\mathrm{R} 70^{\circ}, F_{1,12}=70.342, p<0.001$. Moreover, error $e_{z}$ was statistically significantly different over insertion angles both using the hand $\left(F_{2,24}=12.321, p<0.001\right)$ and the teleoperation system $\left(F_{2,24}=8.878, p=0.001\right)$. Significant pairwise comparison results of this simple main effects analysis are reported in Fig. 11c. Comparing Fig. 11b vs. Fig. 11c, we can
TABLE III

SUMMARY OF EXPERIMENT \#2: STIFFNESS DISCRIMINATION - NEEDLE INSERTION

\begin{tabular}{|c|c|c|}
\hline Subjects & \multicolumn{2}{|l|}{13 (9 males, 4 females) } \\
\hline Task & \multicolumn{2}{|c|}{$\begin{array}{l}\text { Inserting a needle inside two different soft tissue phantoms piled } \\
\text { up and stopping the insertion as soon as a change in stiffness is } \\
\text { perceived }\end{array}$} \\
\hline \multirow[t]{4}{*}{ Conditions } & \multicolumn{2}{|l|}{ Direct hand interaction $(\mathrm{H})$} \\
\hline & \multicolumn{2}{|c|}{ Robotic teleoperation at a force magnification factor of $5(\mathrm{R} 5 \times)$} \\
\hline & \multicolumn{2}{|c|}{ Robotic teleoperation at a force magnification factor of $10(\mathrm{R} 10 \times)$} \\
\hline & \multicolumn{2}{|c|}{ Robotic teleoperation at a force magnification factor of $15(\mathrm{R} 15 \times)$} \\
\hline \multicolumn{3}{|c|}{ Motion scaling factor $\zeta \quad 0.3$} \\
\hline \multicolumn{3}{|c|}{ Best conditions (average \pm standard deviation) } \\
\hline \multicolumn{2}{|c|}{ Penetration error } & $\mathrm{R} 15 \times:(2.6 \pm 1.4) \mathrm{mm}$ \\
\hline \multicolumn{2}{|c|}{ Perceived effectiveness (1-10 range) } & $\mathrm{R} 15 \times: 8.3 \pm 0.6$ \\
\hline \multicolumn{3}{|c|}{ Worst conditions (average \pm standard deviation) } \\
\hline \multicolumn{2}{|c|}{ Penetration error } & $\mathrm{H}:(10.7 \pm 2.0) \mathrm{mm}$ \\
\hline \multicolumn{2}{|c|}{ Perceived effectiveness (1-10 range) } & $\mathrm{H}: 1.5 \pm 0.6$ \\
\hline \multicolumn{3}{|c|}{ Statistical analysis (repeated-measures ANOVA, $a=0.05$ ) } \\
\hline \multicolumn{3}{|c|}{ Penetration error (significant $p$ values only) } \\
\hline \multicolumn{2}{|r|}{$\mathrm{H}$ vs. $\mathrm{R} 5 \times$} & $p=0.002$ \\
\hline \multicolumn{2}{|r|}{$\mathrm{H}$ vs. $\mathrm{R} 10 \times$} & $p<0.001$ \\
\hline \multicolumn{2}{|r|}{ H vs. $\mathrm{R} 15 \times$} & $p<0.001$ \\
\hline \multicolumn{2}{|r|}{$\mathrm{R} 5 \times$ vs. $\mathrm{R} 10 \times$} & $p=0.015$ \\
\hline \multicolumn{2}{|r|}{ R5 $\times$ vs. $\mathrm{R} 15 \times$} & $p=0.007$ \\
\hline \multicolumn{2}{|r|}{$\mathrm{R} 10 \times$ vs. $\mathrm{R} 15 \times$} & $p=0.041$ \\
\hline \multicolumn{3}{|c|}{ Statistical analysis (related-samples Wilcoxon signed rank, $a=0.05$ ) } \\
\hline \multicolumn{3}{|c|}{ Perceived effectiveness (significant $p$ values only) } \\
\hline \multicolumn{2}{|r|}{$\mathrm{H}$ vs. $\mathrm{R} 5 \times$} & $p=0.002$ \\
\hline \multicolumn{2}{|r|}{$\mathrm{H}$ vs. $\mathrm{R} 10 \times$} & $p=0.001$ \\
\hline \multicolumn{2}{|r|}{ H vs. $\mathrm{R} 15 \times$} & $p<0.001$ \\
\hline \multicolumn{2}{|r|}{ R5 × vs. R15× } & $p=0.001$ \\
\hline
\end{tabular}

see that the total penetration error $e_{p}$ remains constant across insertion angles, while its projection on the normal direction $e_{z}$ increases as the needle angle increases. This may indicate that human users need to penetrate the same amount of tissue to appreciate a difference in stiffness, regardless of the needle angle.

Similarly as before, immediately after the experiment, subjects were asked to report the effectiveness of each feedback condition in completing the given task using bipolar Likerttype nine-point scales. Fig. 11d shows the perceived effectiveness for the two feedback conditions $\mathrm{H}$ and $\mathrm{R}$. A relatedsamples Wilcoxon signed rank test showed a statistically significant difference between the means of the two feedback conditions ( $U=91, p=0.001, a=0.05)$.

All thirteen subjects found the teleoperated condition to be the most effective at letting them keep the desired orientation of the needle shaft and stopping the insertion when entering the bottom phantom. 


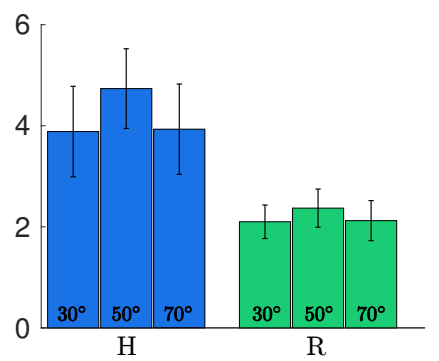

(a) Orientation Error $e_{o}$ (deg).

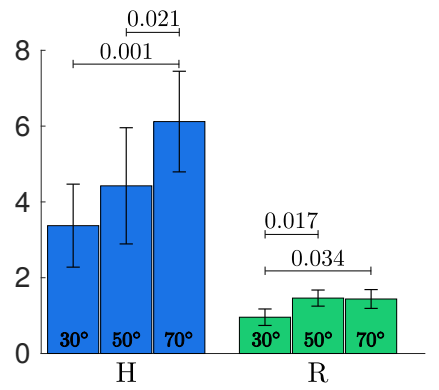

(c) Penetration error $e_{z}(\mathrm{~mm})$.

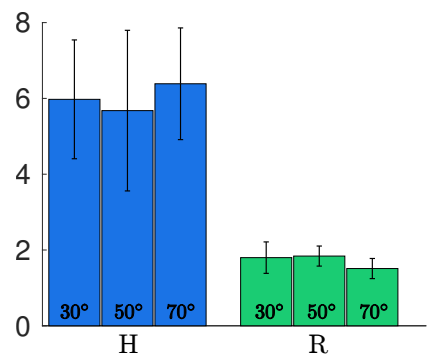

(b) Penetration Error $e_{p}(\mathrm{~mm})$

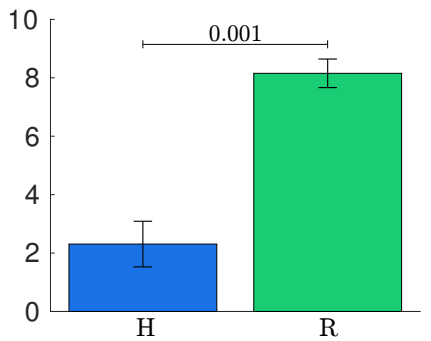

(d) Perceived Effectiveness.

Fig. 11. Experiment \#3: needle insertion along a predetermined direction. Mean and 95\% confidence interval of (a) the insertion orientation error, (b) the total insertion penetration error, (c) its projection along the $z$-direction, and (d) the perceived effectiveness are plotted when the task was performed by hand $(\mathrm{H})$ or by using the robot $(\mathrm{R})$. Four target needle angles were considered: $30^{\circ}, 50^{\circ}$, and $70^{\circ}$. Lower values indicate better performance except for the perceived effectiveness plot.

Experimental conditions, task, and results of this experiment are summarized in Tab. IV.

\section{DISCUSSION}

This paper presents an innovative teleoperation system with magnified haptic feedback for robot-assisted medical procedures. It enables a clinician to intuitively and accurately control the motion of the the slave end-effector while providing him or her with magnified haptic feedback about the interaction with the operating environment. The system aims at improving the haptic perception capabilities of the operating clinician while guaranteeing the safety of the surgical system. We evaluated the proposed approach in three paradigmatic experiments. The first experiment considered a stiffness discrimination task during tool-mediated palpation of soft tissue; the second experiment considered a stiffness discrimination task during needle insertion in soft tissue; and the third experiment evaluated the system in following a predetermined direction when inserting a needle in soft tissue. Providing magnified haptic feedback and guidance significantly improved tasks performance in all the considered experiments. We registered an improvement with respect to direct hand interaction of $80 \%, 306 \%$, and $27 \%$ in stiffness discrimination through palpation, stiffness discrimination during needle insertion, and guidance, respectively. Moreover, all the subjects found conditions employing the teleoperation system to be the most effective at letting them accomplish the proposed task. Finally, the enforced passivity control approach always guaranteed the stability and safety of the system, even in the case of high force

TABLE IV

SUMMARY OF EXPERIMENT \#3: NEEDLE INSERTION ALONG A PREDETERMINED DIRECTION

\begin{tabular}{ll}
\hline Subjects & 13 (9 males, 4 females) \\
\hline Task & $\begin{array}{l}\text { Inserting a needle in soft tissue while maintaining a predetermined } \\
\text { inclination and stopping the insertion when a predetermined depth } \\
\text { is reached }\end{array}$ \\
\hline Conditions & Direct hand interaction (H) \\
& $30^{\circ}, 50^{\circ}$, and $70^{\circ}$ as target needle angles \\
& Robotic teleoperation at a force magnification factor of $10(\mathrm{R})$ \\
$30^{\circ}, 50^{\circ}$, and $70^{\circ}$ as target needle angles
\end{tabular}

Motion scaling factor $\zeta \quad 0.3$

Best conditions (average \pm standard deviation)

$\begin{array}{ll}\text { Orientation error } & \text { R: }(2.2 \pm 0.6) \mathrm{deg} \\ \text { Penetration error } e_{p} & \text { R: }(1.7 \pm 0.5) \mathrm{mm} \\ \text { Penetration error } e_{z} & \text { R: }(1.2 \pm 0.4) \mathrm{mm} \\ \text { Perceived effectiveness } & \text { R: } 8.1 \pm 0.8\end{array}$

Worst conditions (average \pm standard deviation)
Orientation error
$\mathrm{H}:(4.3 \pm 1.5) \mathrm{deg}$
Penetration error $e_{p}$
$\mathrm{H}:(6.0 \pm 3.2) \mathrm{mm}$
Penetration error $e_{z}$
$\mathrm{H}:(4.6 \pm 2.4) \mathrm{mm}$
Perceived effectiveness
$\mathrm{H}: 2.3 \pm 1.4$

Statistical analysis (two-way repeated-measure ANOVA, $a=0.05$ )

\section{Orientation error}

$$
\begin{array}{ll}
\text { Experimental setup * Orientation angle } & \begin{array}{l}
p=0.658 \\
\text { (not significant) }
\end{array} \\
\text { Main effect of experimental setup } & \\
\text { H vs. } \mathrm{R} & p<0.001 \\
\text { Main effect of insertion angle } & \\
30^{\circ} \text { vs. } 50^{\circ} \text { vs. } 70^{\circ} & \begin{array}{l}
p=0.169 \\
\text { (not significant) }
\end{array}
\end{array}
$$

Penetration error $e_{p}$

$$
\begin{array}{ll}
\text { Experimental setup * Orientation angle } & p=0.062 \\
& \text { (not significant) }
\end{array}
$$

Main effects of experimental setup

$$
\mathrm{H} \text { vs. } \mathrm{R} \quad p<0.001
$$

Main effects of insertion angle

$$
\begin{array}{ll}
30^{\circ} \text { vs. } 50^{\circ} \text { vs. } 70^{\circ} & p=0.853 \\
\text { (not significant) }
\end{array}
$$

Penetration error $e_{z}$

$$
\begin{array}{cc}
\text { Experimental setup * Orientation angle } & p=0.043 \\
\text { Simple main effects of experimental setup } & \\
\mathrm{H} 30^{\circ} \text { vs. } \mathrm{R} 30^{\circ} & p<0.001 \\
\mathrm{H} 50^{\circ} \text { vs. } \mathrm{R} 50^{\circ} & p<0.001 \\
\mathrm{H} 70^{\circ} \text { vs. } \mathrm{R} 70^{\circ} & p<0.001 \\
\text { Simple main effects of insertion angle } & \\
\mathrm{H} 30^{\circ} \text { vs. } \mathrm{H} 50^{\circ} \text { vs. } \mathrm{H} 70^{\circ} & p<0.001 \\
\mathrm{R} 30^{\circ} \text { vs. } \mathrm{R} 50^{\circ} \text { vs. } \mathrm{R} 70^{\circ} & p=0.001
\end{array}
$$

Statistical analysis (related-samples Wilcoxon signed rank, $a=0.05$ )

Perceived effectiveness

$$
\mathrm{H} \text { vs. } \mathrm{R}
$$

$p=0.001$ 
magnification rates. Although a higher force magnification rate always led to higher performance, the difference between magnification rates $10 \times$ and $15 \times$ was not as evident as the difference between magnification rates $5 \times$ and $10 \times$. For this reason, given the safety issues arising when increasing the force magnification, it may be not necessary to employ magnification rates as high as $15 \times$. Further psychophysical experiments are needed to assess optimal magnification rates for each task.

In the future, we plan to run a new human-subject study enrolling both novices and experienced clinicians. This study will let us better understand the importance of magnified haptic feedback at various levels of medical experience, in addition to its role in the clinician's learning process. Moreover, we plan to evaluate the proposed teleoperation system in other related tasks, such as biopsy and blunt dissection, and to study the possible perceptual interactions between kinesthetic haptic feedback and other types of haptic feedback, such as vibrotactile and contact deformation feedback. We will also test whether palpation sensations could be conveyed more effectively using alternative end-effectors, we will study how to extend the capabilities of the proposed guidance haptic system to steer flexible thin needles, and we will take into account the effects of needle deformations during the insertion. Finally, we intend to investigate the practical translational aspects of the proposed robotic system by applying the same control and haptic rendering techniques to commercially available surgical robots, such as the Raven or the da Vinci Surgical System.

\section{FUNDING}

The research leading to these results has received funding from the European Union Seventh Framework Programme FP7/2007-2013 under grant agreement $n^{\circ} 601165$ of the project "WEARHAP - WEARable HAPtics for humans and robots".

\section{REFERENCES}

1. Lanfranco A. R, Castellanos A. E, Desai J. P, Meyers W. C. Robotic surgery: a current perspective Ann Surg. 2004;239:14-21.

2. Herron D, Marohn M, others . A consensus document on robotic surgery Surg Endosc. 2008;22:313-325.

3. Salcudean S. E, Ku S, Bell G. Performance measurement in scaled teleoperation for microsurgery in Proc. First Joint Conference on Computer Vision, Virtual Reality and Robotics in Medicine and Medial Robotics and ComputerAssisted Surgery:789-798 1997.

4. Abayazid M, Pacchierotti C, Moreira P, Alterovitz R, Prattichizzo D, Misra S. Experimental evaluation of comanipulated ultrasound-guided flexible needle steering Int J Med Robot. 2016;12:219-230.

5. Kazi A. Operator performance in surgical telemanipulation Presence Teleop Virt. 2001;10:495-510.

6. Moody L, Baber C, Arvanitis T. N, others . Objective surgical performance evaluation based on haptic feedback :304-310 IOS Press 2002.

7. Pacchierotti C, Prattichizzo D, Kuchenbecker K. J. Cutaneous feedback of fingertip deformation and vibration for palpation in robotic surgery IEEE Trans Biomed Eng. 2016;63:278-287.

8. Kennedy C. W, Hu T, Desai J. P, Wechsler A. S, Kresh J. Y. A novel approach to robotic cardiac surgery using haptics and vision Cardiovasc Eng. 2002;2:15-22.

9. De Lorenzo D, De Momi E, Dyagilev I, et al. Force feedback in a piezoelectric linear actuator for neurosurgery Int J Med Robot. 2011;7:268-275.

10. Tavakoli M, Patel R, Moallem M. Haptic interaction in robot-assisted endoscopic surgery: a sensorized endeffector Int J Med Robot. 2005;1:53-63.

11. Pacchierotti C, Scheggi S, Prattichizzo D, Misra S. Haptic feedback for microrobotics applications: a review Front Robot AI. 2016;3.

12. Pillarisetti A, Pekarev M, Brooks A. D, Desai J. P. Evaluating the effect of force feedback in cell injection IEEE Trans Autom Sci Eng. 2007;4:322-331.

13. Hannaford B, Wood L, McAffee D. A, Zak H. Performance Evaluation of a Six-Axis Generalized ForceReflecting Teleoperator IEEE Trans Syst, Man, Cybern, Syst. 1991;21:620-633.

14. Meli L, Pacchierotti C, Prattichizzo D. Sensory subtraction in robot-assisted surgery: fingertip skin deformation feedback to ensure safety and improve transparency in bimanual haptic interaction IEEE Trans Biomed Eng. 2014;61:1318-1327.

15. Wagner C. R, Stylopoulos N, Howe R. D. The role of force feedback in surgery: analysis of blunt dissection in Proc. 10th Symposium of Haptic Interfaces for Virtual Environment and Teleoperator Systems:68-74 2002.

16. Okamura A. M. Haptic feedback in robot-assisted minimally invasive surgery Curr Opin Urol. 2009;19:102.

17. Pacchierotti C, Prattichizzo D, Kuchenbecker K. J. Displaying sensed tactile cues with a fingertip haptic device IEEE Trans Haptics. 2015;8:384-396.

18. Massimino M. J, Sheridan T. B. Teleoperator performance with varying force and visual feedback Human Factors. 1994;36:145-157.

19. Prattichizzo D, Pacchierotti C, Rosati G. Cutaneous force feedback as a sensory subtraction technique in haptics IEEE Trans Haptics. 2012;5:289-300.

20. Westebring-Van Der Putten E, Goossens R, Jakimowicz J, Dankelman J. Haptics in minimally invasive surgery-a review Minim Invasive Ther Allied Technol. 2008;17:316.

21. Pacchierotti C, Meli L, Chinello F, Malvezzi M, Prattichizzo D. Cutaneous haptic feedback to ensure the stability of robotic teleoperation systems Int J Robot Res. 2015;34:1773-1787.

22. King C.-H, Culjat M. O, Franco M. L, et al. Tactile feedback induces reduced grasping force in robot-assisted surgery IEEE Trans Haptics. 2009;2:103-110.

23. King C.-H, Culjat M. O, Franco M. L, et al. A multielement tactile feedback system for robot-assisted minimally invasive surgery IEEE Trans Haptics. 2009;2:52-56.

24. Li M, Luo S, Seneviratne L, Nanayakkara T, Althoefer K, Dasgupta P. Haptics for Multi-fingered Palpation in Proc. IEEE Int. Conf. on Systems, Man, and Cybernetics:4184- 
41892013.

25. Stanley A, Okamura A. Controllable Surface Haptics via Particle Jamming and Pneumatics IEEE Trans Haptics. 2015;8:20-30.

26. Li M, Ranzani T, Sareh S, et al. Multi-fingered haptic palpation utilizing granular jamming stiffness feedback actuators Smart Mater Struct. 2014;23:095007.

27. Gerovich O, Marayong P, Okamura A. M. The effect of visual and haptic feedback on computer-assisted needle insertion Comput Aided Surg. 2004;9:243-249.

28. Pacchierotti C, Abayazid M, Misra S, Prattichizzo D. Teleoperation of steerable flexible needles by combining kinesthetic and vibratory feedback IEEE Trans Haptics. 2014;7:551-556.

29. Hashtrudi-Zaad K, Salcudean S. E. Transparency in timedelayed systems and the effect of local force feedback for transparent teleoperation IEEE Trans Robot Autom. 2002;18:108-114.

30. Diolaiti N, Niemeyer G, Barbagli F, Salisbury J. K. Stability of haptic rendering: Discretization, quantization, time delay, and Coulomb effects IEEE Trans Robot. 2006;22:256-268.

31. Bolopion A, Régnier S. A review of haptic feedback teleoperation systems for micromanipulation and microassembly IEEE Trans Autom Sci Eng. 2013;10:496-502.

32. Venture G, Haliyo D. S, Régnier S, Micaelli A. Forcefeedback micromanipulation with unconditionally stable coupling in Proc. IEEE/RSJ Int. Conf. on Intelligent Robots and Systems:1923-1928 2005.

33. Kim S.-G, Sitti M. Task-based and stable telenanomanipulation in a nanoscale virtual environment IEEE Trans Autom Sci Eng. 2006;3:240-247.

34. Schoonmaker R. E, Cao C. G. L. Vibrotactile force feedback system for minimally invasive surgical procedures in Proc. IEEE Int. Conf. on Systems, Man and Cybernetics;3:2464-2469 2006.

35. Griffin W. B, Provancher W. R, Cutkosky M. R. Feedback Strategies for Telemanipulation with Shared Control of Object Handling Forces Presence Teleop Virt. 2005;14:720-731.

36. Kitagawa M, Dokko D, Okamura A. M, Yuh D. D. Effect of sensory substitution on suture-manipulation forces for robotic surgical systems Journal of Thoracic and Cardiovascular Surgery. 2005;129:151-158.

37. Prattichizzo D, Pacchierotti C, Cenci S, Minamizawa K, Rosati G. Using a fingertip tactile device to substitute kinesthetic feedback in haptic interaction Haptics: Generating and Perceiving Tangible Sensations. 2010:125-130.

38. Prattichizzo D, Trinkle J. Chapter 28 on Grasping in Handbook on Robotics (Siciliano B, Kathib O. , eds.):671700 Springer 2008.

39. Franken M, Stramigioli S, Misra S, Secchi C, Macchelli A. Bilateral telemanipulation With time delays: a two-layer approach combining passivity and transparency IEEE Trans Robot. 2011;27:741-756.

40. Lehman C. D, Aikawa T. MR-guided Vacuum-assisted Breast Biopsy: Accuracy of Targeting and Success in Sampling in a Phantom Model 1 Radiology. 2004;232:911-
914.

41. Kobayashi Y, Sato T, Fujie M. G. Modeling of friction force based on relative velocity between liver tissue and needle for needle insertion simulation in Proc. IEEE Int. Conf. of Engineering in Medicine and Biology Society:5274-5278 2009.

42. Okamura A. M, Simone C, Leary M. Force modeling for needle insertion into soft tissue IEEE Trans Biomed Eng. 2004;51:1707-1716.

43. Majewicz A, Wedlick T. R, Reed K. B, Okamura A. M. Evaluation of robotic needle steering in ex vivo tissue in Proc. IEEE Int. Conf. on Robotics and Automation:206820732010.

44. Li W, Belmont B, Shih A. Design and Manufacture of Polyvinyl Chloride (PVC) Tissue Mimicking Material for Needle Insertion Procedia Manufacturing. 2015;1:866878.

45. Podder T, Clark D, Sherman J, et al. Robotic needle insertion in soft material phantoms: an evaluation of the property of commonly used materials Change. 2005;50:1-93.

46. Wedlick T. R, Okamura A. M. Characterization of robotic needle insertion and rotation in artificial and ex vivo tissues in Proc. IEEE RAS/EMBS Int. Conf. on Biomedical Robotics and Biomechatronics:62-68 2012.

47. Liu H, Li J, Song X, Seneviratne L. D, Althoefer K. Rolling indentation probe for tissue abnormality identification during minimally invasive surgery IEEE Trans Robot. 2011;27:450-460.

48. Hui J. C, Kuchenbecker K. J. Evaluating the BioTac's Ability to Detect and Characterize Lumps in Simulated Tissue in Haptics: Neuroscience, Devices, Modeling, and Applications:295-302 2014.

49. Bowyer S. A, Davies B. L, Baena F. Active constraints/virtual fixtures: A survey IEEE Trans Robot. 2014;30:138-157.

50. Craig J. J. Introduction to robotics: mechanics and control;3. Pearson Prentice Hall Upper Saddle River 2005.

51. Pacchierotti C, Magdanz V, Medina-Sanchez M, G. Schmidt O, Prattichizzo D, Misra S. Intuitive control of self-propelled microjets with haptic feedback Journal of Micro-Bio Robotics. 2015;10:37-53.

52. Peon A. R, Pacchierotti C, Prattichizzo D. Vibrotactile stimuli for augmented haptic feedback in robot-assisted surgery in Proc. IEEE World Haptics Conference:473-478 2013.

53. Jones L. A, Sarter N. B. Tactile displays: Guidance for their design and application Human Factors. 2008;50:90111.

54. Kaczmarek K. A, Webster J. G, Rita P, Tompkins W. J. Electrotactile and vibrotactile displays for sensory substitution systems IEEE Trans Biomed Eng. 1991;38:1-16.

55. Landin N, Romano J. M, McMahan W, Kuchenbecker K. J. Dimensional reduction of high-frequency accelerations for haptic rendering in Haptics: Generating and Perceiving Tangible Sensations:79-86 Springer 2010.

56. Draper N. R, Smith H. Applied regression analysis. John Wiley \& Sons 2014. 